\title{
Talking microbes
}

\author{
Understanding the molecules and mechanisms that microbes use to interact with each other and their \\ environments can lead to better antimicrobial drug design as well as a richer understanding of bacterial \\ physiology, ecology and evolution.
}

The popular press typically describes microorganisms mainly as diseasecausing bugs that should be eliminated using antimicrobial drugs. However, this mindset overlooks both the substantial scientific contributions gleaned from studying microbial biology and our growing understanding that different microorganisms are fundamentally distinct in ways that need to be accounted for in drug discovery efforts. For example, many clinically useful antimicrobial compounds originated from bacteria and other microorganisms. By investigating how these natural products function as signals or metabolites in native environments, we can improve our biological understanding, which is critical for the development of future antimicrobial agents. In this themed issue, we examine the new ideas and technologies that are changing the way we think about bacterial communication, both within microbe communities and with their environments.

Though it has long been known that bacteria and other microorganisms exist in communities, there is a growing appreciation that microbial life within communities can differ markedly from solitary existence. For example, Phelan et al. note that a considerable percentage of bacterial genomes is dedicated to shaping the organisms' habitats and maintaining their ecosystems, representing a major commitment to community and niche (Perspective, p. 26). These authors argue that we are still searching for the microbial 'Rosetta stone' to decode bacterial communication, and that more emphasis should be placed on understanding the functional roles of these gene products and their metabolites in communication.

Understanding the functions of microbial natural products within their native environments is a challenge that the chemical biology community is well placed to take up. Phelan et al. point to nascent technologies such as MS imaging as tools to explore the ecology of microbial communication through monitoring of individual metabolites. Connell, Whitely and Shear suggest that new fabrication technologies will be critical in creating threedimensional microbial environments that faithfully represent microbial niches on a microscopic scale (Commentary, p. 10). These devices can also serve as useful platforms for chemical biologists to explore and manipulate microbial communication-for instance, for creating localized gradients of chemotactic substances, for studying cell migration or for microanalysis of quorum sensing signals. Insights gained from these efforts could be applied to engineer microbes for industrial or medical applications.

New experimental strategies such as those outlined by Phelan et al. and Connell, Whitely and Shear will be particularly important in investigating the vast majority of microbial pathogens that remain inaccessible to genetic manipulation. As these species have evolved elaborate pathogenic mechanisms and equally robust defense mechanisms, new antibiotics are sorely needed. Understanding the metabolites that microbes rely upon to interact with their environments and how pathogens respond to environmental and pharmacological agents can improve prospects for finding new antimicrobials. For example, Roemer et al. highlight genetic approaches that can facilitate the discovery of new antifungal and antibacterial compounds and their targets (Review, p. 46).

Parallel efforts are also required to thwart resistance to available antibiotics. Chait, Vetsigian and Kishony approach this problem from an evolutionary and ecological perspective (Commentary, p. 2). These authors consider how populations of both sensitive and resistant microorganisms are maintained in nature and propose that aspects of the soil environment ensure that full resistance does not occur. They further highlight evidence suggesting that combinations of antibiotics do not always yield anticipated outcomes owing to phenomena such as 'collateral sensitivity', a mechanism that increases sensitivity to certain toxins. Thus, understanding resistance requires an appreciation of the entire environment and its complex network of interactions.

Lee and Collins go on to suggest that a major challenge to the development of new antibiotics is to understand how bacteria work together to manipulate their extracellular environments to survive stressful, unpredictable situations (Commentary, p. 6). For example, they point out that all antibiotics, regardless of their primary target, work through similar cell death pathways that involve stimulation of central metabolism. Indeed, a rich understanding of microbial chemistry and biology at a metabolic level within cells and within communities should lead to fundamental new insights into not only antimicrobial design but also cell-cell and cell-host communication. Along these lines, Ashida et al. remind us that small molecules are not the only molecules involved in these interactions in their description of injection systems that pathogenic bacteria use to communicate with and manipulate their environment during infection of host intestinal cells (Review, p. 36). These viewpoints argue for more realistic recapitulations of host environments in attempts to create new antimicrobial agents.

As we better understand bacterial communities and extend microbiology research to less well-characterized microbes, we are forced to re-examine current assumptions about these organisms. Microbial classification now includes a wider range of bacterial characteristics, such as morphologies, genomes and metabolomes, all of which have refined our understanding of microbial phylogenies (such as the fairly recent splitting of archaeal and bacterial trees). For example, the traditional distinction of Gram-positive versus Gram-negative bacteria was based on differing abilities to take up a particular dye molecule, a characteristic linked to bacterial membrane composition. However, Vollmer suggests that this relatively simplistic classification scheme may cause scientists to overlook important sources of microbial diversity, as illustrated by a case study of Acetonema longum, a recently explored hybrid organism that phylogenetically links to the Gram-positive Firmicutes but stains Gramnegative (Commentary, p. 14).

Microbial communities are complex systems that are regulated by networks of endogenous small molecules and other diverse environmental and exogenous signals. Understanding these networks at the molecular level represents a challenge for chemical biologists but offers great potential for new fundamental insights into microbial biology and ecology while providing the necessary knowledge to manipulate these systems for applications in medicine and synthetic biology. 